\title{
Biochemical and cellular properties of Gluconacetobacter xylinus cultures exposed to different modes of rotating magnetic field
}

\author{
Karol Fijałkowski', Radosław Drozd ${ }^{1}$, Anna Żywicka', Adam F. Junka², Marian Kordas³, \\ Rafal Rakoczy $^{3 *}$
}

${ }^{1}$ West Pomeranian University of Technology, Szczecin, Faculty of Biotechnology and Animal Husbandry, Department of Immunology, Microbiology and Physiological Chemistry, Poland

${ }^{2}$ Medical University of Wroclaw, Department of Pharmaceutical Microbiology and Parasitology, Poland

${ }^{3}$ West Pomeranian University of Technology, Szczecin, Faculty of Chemical Technology and Engineering, Institute of Chemical Engineering and Environmental Protection Processes, Faculty of Chemical Technology and Engineering, Poland *corresponding author: e-mail: rrakoczy@zut.edu.pl

\begin{abstract}
The aim of the present study was to evaluate the impact of a rotating magnetic field (RMF) on cellular and biochemical properties of Gluconacetobacter xylinus during the process of cellulose synthesis by these bacteria. The application of the RMF during bacterial cellulose (BC) production intensified the biochemical processes in G. xylinus as compared to the RMF-unexposed cultures. Moreover, the RMF had a positive impact on the growth of cellulose-producing bacteria. Furthermore, the application of RMF did not increase the number of mutants unable to produce cellulose. In terms of $\mathrm{BC}$ production efficacy, the most favorable properties were found in the setting where RMF generator was switched off for the first $72 \mathrm{~h}$ of cultivation and switched on for the further 72 $\mathrm{h}$. The results obtained can be used in subsequent studies concerning the optimization of $\mathrm{BC}$ production using different types of magnetic fields including RMF, especially.
\end{abstract}

Keywords: bacterial cellulose; bioprocess; Gluconacetobacter xylinus; rotating magnetic field; optimization.

\section{INTRODUCTION}

Bacterial cellulose (BC) is a type of natural cellulose synthesized in abundance by Gluconacetobacter xylinus. Although chemically identical to plant cellulose, BC is characterized by high purity and a unique nanostructure. These features determine its distinguished physical and mechanical properties such as high porosity, high tensile strength, high water binding capacity and good biocompatibility ${ }^{1}$. The bacteria with an ability to synthesize cellulose developed specialized metabolic pathways responsible for the conversion of glucose monomers into polymeric cellulose. This multi-step process, involving a number of enzymes, regulatory proteins, catalyst complexes and cofactors, is accurately and specifically controlled ${ }^{2}$.

Glucose is the primary substrate for the synthesis of cellulose. It is incorporated in a 4-step metabolic pathway placed in cytosol and converted to UDP-glucose. This reaction is catalyzed by uridine-diphosphate-glucose pyrophosphorylase (EC 2.7.7.64, UDP-glucose pyropho-

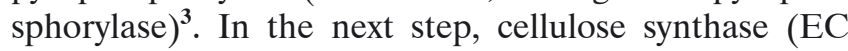
2.4.1.12) located in the bacterial cell wall catalyzes the polymerization of UDP-glucose to poly $\beta-1-4$ glucan ${ }^{4}$. Cyclo-3,6':3',6-diguanidine monophosphate (c-di-GMP) is the essential cofactor for this reaction, responsible for the activation of the synthase. The process of polymerization of UDP-glucose to poly $\beta-1-4$ glucan can be disrupted by such environmental conditions as oxygenation, $\mathrm{pH}$ or temperature ${ }^{5}$.

Glucose can also be readily converted to gluconic acid which significantly affects the $\mathrm{pH}$ of the medium. This may adversely affect the efficiency of the $\mathrm{BC}$ synthesis process. However, gluconic acid may be re-converted to glucose-1-phosphate (G1P) and thus it can also be used for cellulose synthesis. This pathway starts from the gluconate permease-assisted transfer of gluconate into cytosol. Then the gluconic acid is converted to 6-phospho-gluconate, which is incorporated into pentose phosphate pathway (PP), from which it is transferred to Embden-Meyerhof-Parnas (EMP, glycolysis) pathway and finally converted to glucose-1-phosphate which can be used for UDP-glucose synthesis. ${ }^{6}$. The efficiency of these pathways depends on the availability of oxygen. This element is also a factor affecting the rate of transformation of tricarboxylic acid cycle (TCA), whose metabolites affect the activity of enzymes in the EMP and PP pathways, and thus the amount of the cellulose produced ${ }^{7}$.

A standard medium for the production of cellulose contains ethanol. This alcohol is a substrate for alcohol dehydrogenase which converts it to acetaldehyde. The product of this reaction is then oxidized by acetaldehyde dehydrogenase to acetate and incorporated into the tricarboxylic acid cycle. ATP produced in the respiratory pathway is a competitive inhibitor for glucose-6-phosphate dehydrogenase $(\mathrm{G} 6 \mathrm{PD})^{8}$. This enzyme is responsible for the conversion of G6P to 6-phospho gluconate and finally for the exclusion of glucose molecules from the pool available for direct synthesis of cellulose. Like ethanol, the acetic acid which is formed during the fermentation may be used also for the production of ATP, thus increasing the yield of cellulose production'.

There are two main methods of $\mathrm{BC}$ production: static cultivation, which results in the accumulation of a gelatinous membrane of cellulose on the surface of the medium, and shaking/agitated culture, where cellulose is synthesized in deep media in the form of fibrous suspensions, pellets, or irregular masses ${ }^{\mathbf{1 0}}$. The production of $\mathrm{BC}$ by static cultivation requires a long culture period (10 days to 6 weeks) and is labour-consuming, thus resulting in a low productivity. Earlier reports also showed that insufficient supply of substrate (e.g. glucose) to the bacteria may be the external factor limiting the yield of synthesized $\mathrm{BC}^{\mathbf{1 1}}$. These considerations have resulted in the development of new methods of $\mathrm{BC}$ production ${ }^{12-13}$. 
The development of a new method of BC production was also the aim of our previous study in which we described the potential of RMF-assisted bioreactor for the biosynthesis of this polymer ${ }^{14}$. Based on the results obtained, we assumed that if we succeed in determining the appropriate conditions of RMF exposure, we would be able to reduce the problems with the rate of mass transfer limitation, especially insufficient substrate supply. We also hypothesized that our invention would lead to the enrichment of by-products yield during the $\mathrm{BC}$ production process and to more efficient use of nutrients (mainly glucose). Therefore, in the present study we investigated whether the RMF, depending on the mode of exposure, may have an impact on the biochemical and cellular properties of $G$. xylinus during $\mathrm{BC}$ biosynthesis. The analyzed biochemical parameters included glucose utilization as well as gluconic and acetic acid production. The growth rate of G. xylinus cells present in the culture medium and embedded within $\mathrm{BC}$ as well as generation of mutants unable to produce cellulose was also monitored.

\section{MATERIAL AND METHODS}

\section{Experimental set-up}

The experiment was performed using a self-designed RMF exposure system adapted for biological studies, described in more detailed manner in our previous work $^{\mathbf{1 4}}$. For the purpose of this study, we used an improved version of the magnetic field bioreactor, in which the gap between the windings of RMF generator and the cylindrical glass container with the tested samples was reduced. Therefore, the diameter of the container placed inside the RMF generator was higher. It allowed to place the probes with the culture medium in the area of the minimal RMF variability and harmonize the dose of magnetic field absorbed by the exposed cells. Similarly to our previous research, the RMF was characterized by a mean value of magnetic induction $(B)$ equals 34 $\mathrm{mT}$ (the maximum magnetic induction which could be achieved in the bioreactor). Figure 1 presents a photo of the RMF generator and a diagram showing its axial section with an indicated location of the culture tubes.

\section{Microorganisms and culture conditions}

The 7-day culture of Gluconacetobacter xylinus (Deutsche Sammlung von Mikroorganismen und Zellkulturen - DSM 46604) was shaken and $100 \mu \mathrm{L}$ of bacterial su-

a)

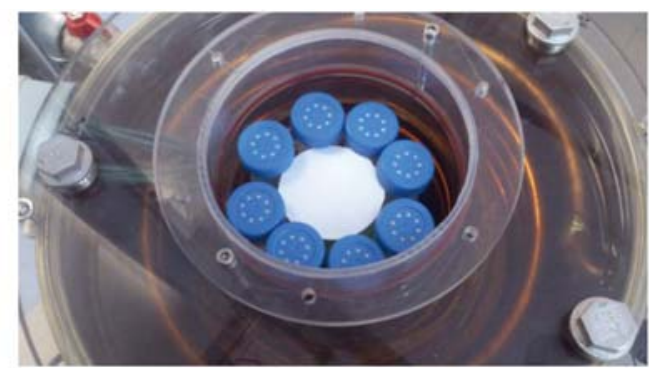

b)

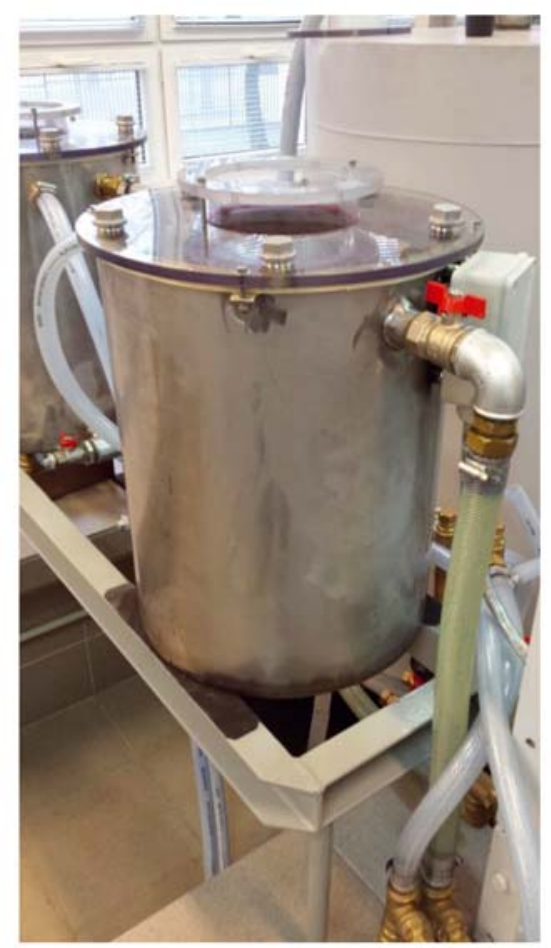

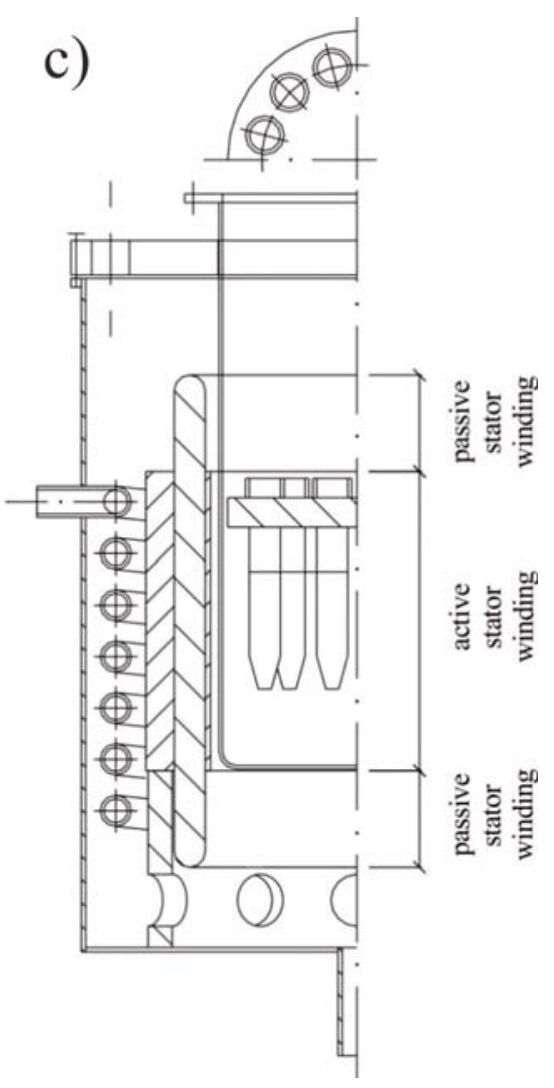

Figure 1. RMF generator $(a, b)$ and its axial section with indicated location of the culture tubes (c) 
spension was transferred to $25 \mathrm{~mL}$ a Herstin-Schramm (H-S) medium in $50 \mathrm{~mL}$ plastic tubes with caps having holes covered with capillary pore filter membrane (pore size of $0.2 \mu \mathrm{m}$ ), which allowed to maintain aerobic conditions during exposure while maintaining sterility (CELLSTAR $^{\circledR}$ CELLreactor $^{\mathrm{TM}}$, Polypropylene Filter Top Tube, Greiner Bio-One, USA). The exposure to the $\mathrm{RMF}$ during the $\mathrm{BC}$ synthesis was conducted in 3 different modes:

G. xylinus cultures were cultivated for $72 \mathrm{~h}$ under constant exposure to the RMF ( $f=50 \mathrm{~Hz}, B=34 \mathrm{mT}, 28^{\circ} \mathrm{C}$ incubation temperature) followed by $72 \mathrm{~h}$ of incubation under the same conditions but with the RMF generator switched off ( $B=0 \mathrm{mT}, 28^{\circ} \mathrm{C}$ incubation temperature).

G. xylinus cultures were cultivated for $72 \mathrm{~h}$ in the RMF generator which was switched off $\left(B=0 \mathrm{mT}, 28^{\circ} \mathrm{C}\right.$ incubation temperature) and then RMF generator was switched on and cultivation was carried out for further $72 \mathrm{~h}$ at the same conditions but under constant exposure to the RMF, $\left(f=50 \mathrm{~Hz}, B=34 \mathrm{mT}, 28^{\circ} \mathrm{C}\right.$ incubation temperature).

G. xylinus cultures were cultivated for $144 \mathrm{~h}$ under constant exposure to the RMF ( $f=50 \mathrm{~Hz}, B=34 \mathrm{mT}$, $28^{\circ} \mathrm{C}$ incubation temperature).

For control purposes, G. xylinus cultures were cultivated for the same time and under the same conditions but without exposition to the RMF. The control samples were incubated in the water bath to maintain the temperature of the test tubes in the RMF generator. The fluctuation of temperature was the same for RMF-exposed and control samples, with deviation less than $0.5^{\circ} \mathrm{C}$. It was confirmed using - Hall probe (Smart Magnetic Sensor-102, Asonik, Poland) that the source of the RMF did not have an impact on the control samples during the experiment $(B \leq 0.05 \mathrm{mT})$.

\section{Biochemical analysis}

All biochemical measurements were performed using commercial kits according to manufacturer instructions. The concentration of glucose in the culture medium was determined enzymatically using the Glucose Assay Kit (BioMaxima, Poland) according to the manufacturer's protocol. The results were expressed as \% of utilized glucose calculated by the formula:

$\%$ of utilized glucose $=\left(1-\frac{\mathrm{mmol} \cdot 1^{-1} \text { of glucose in culture media }}{\mathrm{mmol} \cdot 1^{-1} \text { of glucose in media without cells }}\right) 100 \%$

The gluconic acid content in the culture medium was measured using K-GATE kit (Megazyme Inc. Irleand) according to the manufacturer's instructions and expressed in $\mathrm{g} / \mathrm{L}$.

The acetic acid concentration in the culture medium was measured using K-ACETRM kit (Megazyme Inc. Irleand) according to the manufacturer's instructions and expressed in $\mathrm{mg} / \mathrm{L}$.

The percentage of efficiency ratio of $\mathrm{BC}$ production process was calculated using the formula:

$\% \mathrm{BC}$ efficiency ratio $=\frac{\text { mass of dried } \mathrm{BC}[\mathrm{mg}]}{\text { initialmass of glucose }[\mathrm{mg}]-\text { mgresidual glucose }[\mathrm{mg}]} 100 \%$
Quantification of cellulose producing bacteria and determination of non-cellulose-producing mutants

The density and viability of BC-producing bacteria were determined twice. For the first time in liquid $\mathrm{H}-\mathrm{S}$ medium, after cellulose pellicle removal from the culture tube and for the second time after digestion of the removed cellulose pellicle with cellulose. For the digestion, the cellulose pellicles were washed in distilled water, transferred to $5 \mathrm{~mL}$ of the solution of cellulase (Sigma-Aldrich, Sigma-Aldrich, Germany) in 0.05 mo$1 / \mathrm{L}$ citrate buffer $(\mathrm{pH} 4.8)$ and incubated with shaking for $24 \mathrm{~h}$ at $35^{\circ} \mathrm{C}$. Then, the samples consisting of the culture medium or suspension obtained after digestion with cellulase were centrifuged for $20 \mathrm{~min}$ at $3.300 \mathrm{x} \mathrm{g}$. The obtained pellets were washed in PBS (Phosphate Buffered Saline, Sigma-Aldrich), centrifuged at $3.300 \mathrm{x} \mathrm{g}$ for $20 \mathrm{~min}$ and restored to the original volume with PBS.

The number of microorganisms was determined by performing quantitative plating on Petri dishes containing $\mathrm{H}-\mathrm{S}$ microbiological medium. The H-S medium was subsequently incubated for $72 \mathrm{~h}$ at $28^{\circ} \mathrm{C}$. After incubation, the grown colonies were counted and the number of CFU (Colony Forming Units) per $1 \mathrm{~g}$ of cellulose or per $1 \mathrm{~mL}$ of the medium, taking into account the volume of the buffer used for making bacterial suspension and the volume of suspension spread on the Petri dishes, was determined.

To determine cellulose non-producing mutants, the Petri dishes containing $\mathrm{H}-\mathrm{S}$ agar medium with grown colonies was flooded with $20 \mathrm{~mL}$ of the solution of PBS with $0.01 \%$ of Tinopal LPW dye (Calcofluor White M2R, Tinopal UNPA-GX, Sigma-Aldrich) and incubated for 24 $\mathrm{h}$ in darkness to let the dye stain the cellulose synthesized by the bacteria around colonies. Next, the colonies were examined under longwave UV light (UV Cabinet, CAMAG, Switzerland). Filamentous luminescent zones were observed around the cellulose-producing colonies. The above image was not observed in the case of cellulose non-producing mutants. The cellulose producing and non-producing colonies were counted and the number of CFU per $1 \mathrm{~g}$ of cellulose or per $1 \mathrm{~mL}$ medium was determined.

\section{Statistical analysis}

The data are presented as the means \pm standard errors of the means (SEM) calculated from five measurements. To compare different experiments, the results obtained for RMF-exposed samples were normalized in relation to control samples and compared with each other. The statistical significance of the differences between the experimental and the control samples were analyzed using Student's t-test. The statistical differences between various modes of RMF exposure were determined by one-way analysis of variance (ANOVA). All the analyses were considered statistically significant when the P-value was less than 0.05 .

\section{RESULTS}

\section{Biochemical analysis}

The results of the present study showed that the RMF, depending on the exposure method applied, exerted 
various effects on the biochemical parameters analyzed (Fig. 2a, 2b, 2c). The highest glucose consumption and production of both acids in comparison to the unexposed controls was observed in the second mode of exposure in which G. xylinus was cultivated for $72 \mathrm{~h}$ in the switched off $\left(B=0 \mathrm{mT}\right.$, incubation temperature $\left.28^{\circ} \mathrm{C}\right) \mathrm{RMF}$ generator and then for further $72 \mathrm{~h}$ under the same conditions but under constant exposure to the RMF ( $B$ $=34 \mathrm{mT}$, incubation temperature $28^{\circ} \mathrm{C}$ ). In this case, the difference between glucose consumption, acetic acid and gluconic acid production assayed for the RMF-exposed samples and unexposed controls were $67 \%, 19 \%$ and $21 \%$ respectively. Similar results were found for cultures exposed to the first mode of exposure. In this case, the differences in glucose consumption and acetic acid production were only slightly lower in comparison with the second mode of exposure. However, the gluconic acid production in the second mode of exposure was at a significantly higher level as compared to the first mode of exposure. In the cultures exposed to the first and second modes, acetic acid production was slightly, however statistically significantly, higher in comparison

a)

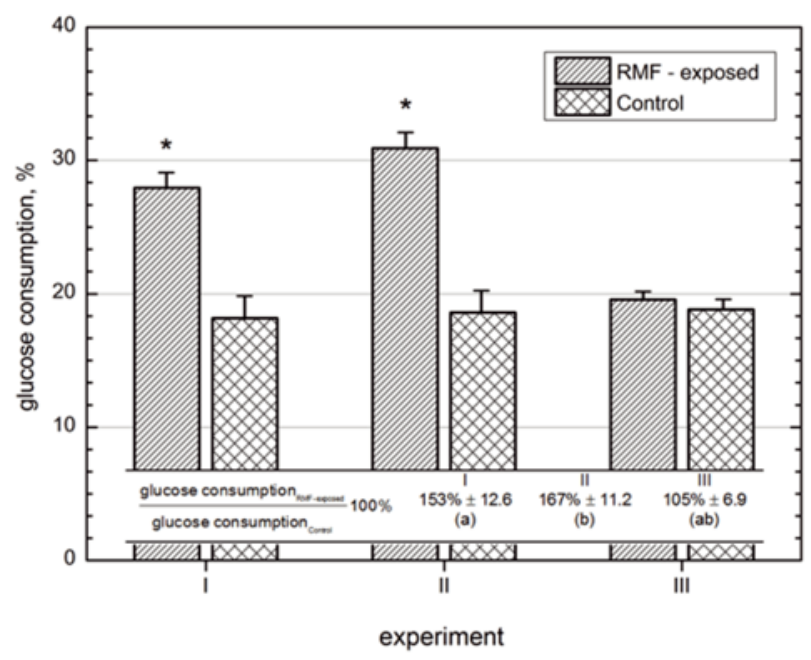

c)

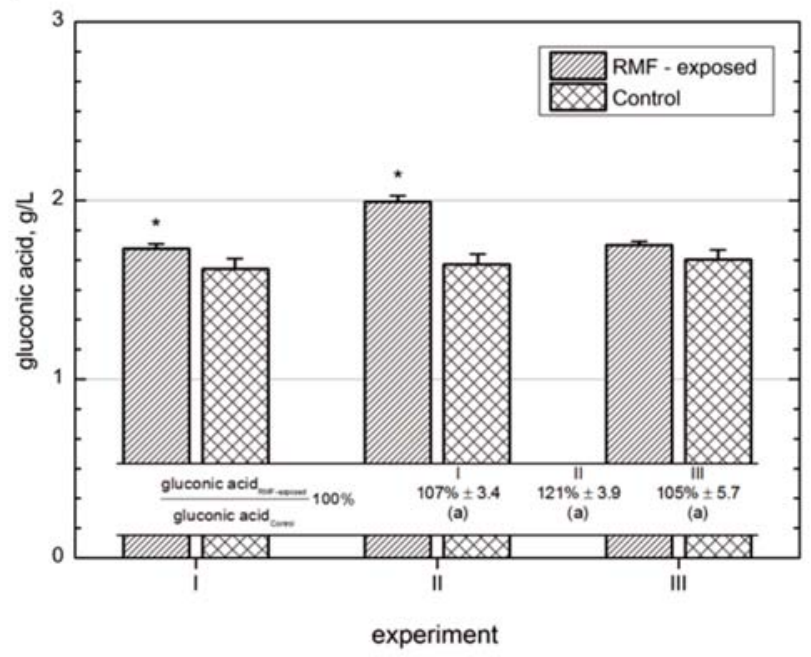

to the third mode. The constant exposure of G. xylinus cultures to RMF for 6 days resulted in decreased biochemical properties in comparison to the experiment in which the exposure was carried out only for a half of this time. It was also found that regardless of the mode of exposure and of the results of biochemical parameters, the $\mathrm{pH}$ values were at a similar level in all cultures (Fig. 3).

The changes in biochemical parameters correlated with the efficiency of the BC production process (Fig. 4). Constant exposure to RMF for 6 days (experiment III) resulted in lower percentages of efficiency values as compared to the experiments in which exposure was carried out for 3 days (experiments I and II). Moreover, the highest efficiency values were observed for cultures cultivated in the first and second modes of exposure in which G. xylinus was cultivated alternately for $72 \mathrm{~h}$ in a RMF generator which was switched off $(B=0$, incubation temperature $28^{\circ} \mathrm{C}$ ) and for $72 \mathrm{~h}$ under the same conditions but with constant exposure to $\mathrm{RMF}$ $\left(B=34 \mathrm{mT}\right.$, incubation temperature $\left.28^{\circ} \mathrm{C}\right)$.

b)

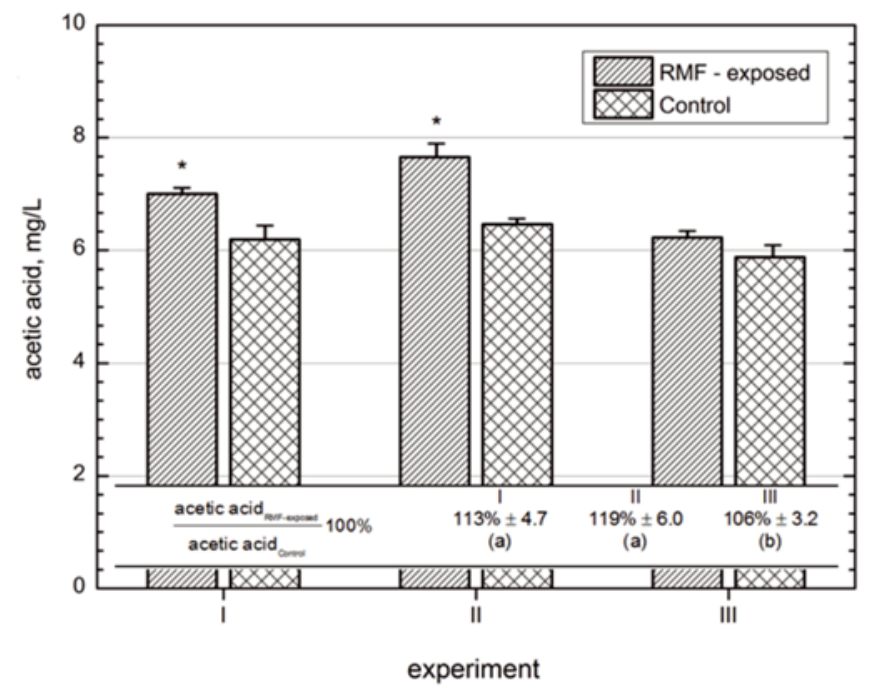

Figure 2. The biochemical parameters of G. xylinus cultures; (a) glucose consumption, (b) acetic acid production, (c) gluconic acid production. Data are presented as a mean \pm standard error of the mean (SEM). * - statistically significant difference between RMF-exposed cultures and control cultures; mean values with different letters $(\mathrm{a} / \mathrm{b} / \mathrm{c})$ are significantly different. The differences were considered statistically significant when the P-value was less than 0.05 


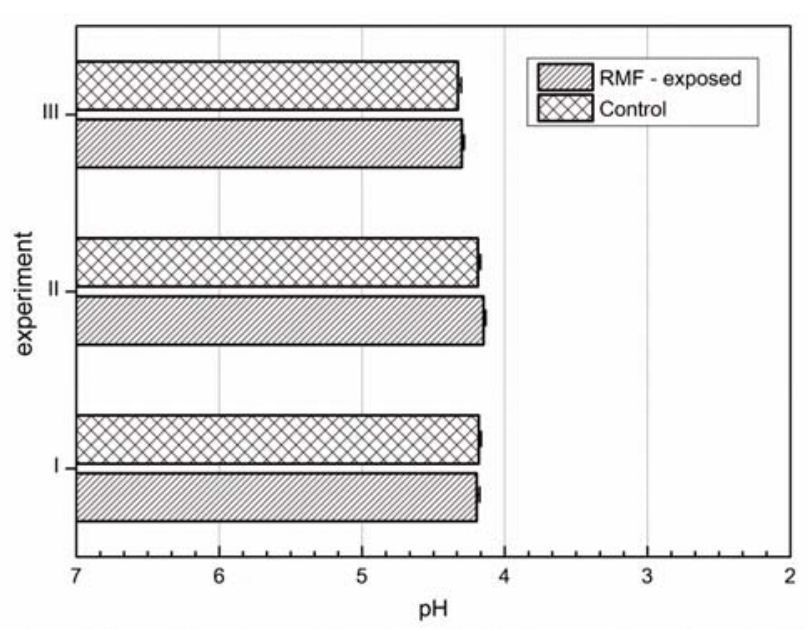

Figure 3. The $\mathrm{pH}$ values of $G$. xylinus cultures

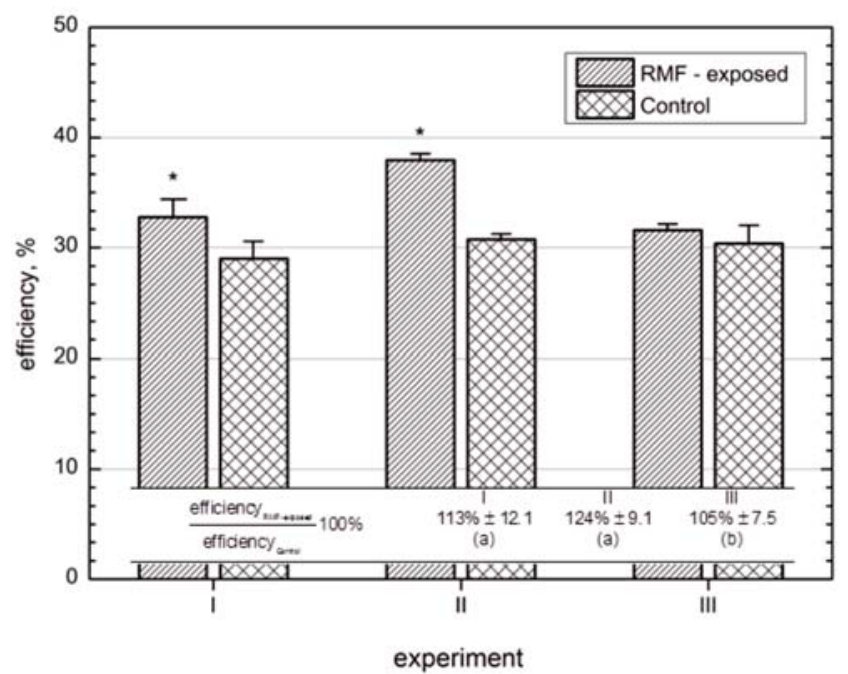

Figure 4. The efficiency of the $\mathrm{BC}$ production process. Data are presented as a mean \pm standard error of the mean (SEM). * - statistically significant difference between RMF-exposed cultures and control cultures; mean values with different letters $(\mathrm{a} / \mathrm{b})$ are significantly different. The differences were considered statistically significant when the P-value was less than 0.05

Analysis of cellulose producing bacteria and cellulose non-producing mutants

In the study of the cellular parameters it was found that RMF, regardless of the exposure method, did not induce of the formation of cellulose non-producing mutants (Table 1). In any case, the number of G. xylinus colonies which were unable to synthesize cellulose did not exceed 20, which accounted for approximately $0.0001 \%$ of all $G$. xylinus cells present in the culture.
In case of the bacterial cells recovered from the cellulose pellicles it was revealed that RMF, regardless of the exposure method, caused a statistically significant increase in CFU number as compared to the unexposed controls. However, the strongest impact on the $G$. xylinus cell number attached to cellulose ribbons was found for the second (II) mode of exposure. In this case, exposure to RMF caused a $43 \%$ increase in cell number as compared to the control samples. Contrary, the lowest effect of RMF exposure was observed for the third (III) mode of exposure. Such variation in CFU was not observed for the bacterial cells released from the cellulose pellicle and present in the medium used for cultivation of G. xylinus during the experiment. In this case, only the second (II) mode of exposure caused a significant increase in the number of CFU compared to the unexposed control samples.

\section{DISCUSSION}

The use of different types of magnetic fields (MFs) is an innovative engineering approach that may find application in various biotechnological processes, including the production of cellulose by bacteria ${ }^{14-15}$. There are numerous reports showing that MFs may have influence on microorganisms and thus change their biotechnological potential $^{16-18}$. The research concerning the use of MFs in the biotechnological engineering conducted to date have concerned mostly static magnetic fields (SMFs), whereas the biotechnologically promising idea to use rotating magnetic fields (RMFs) is developed just relatively recently ${ }^{19-21}$. In previous studies we showed that the RMF can be used to increase (over 20\% compared to the bacteria non-exposed to RMF) the growth and cell metabolic activity of such microorganisms as Staphylococcus aureus, Escherichia coli, Serratia marcescens, Streptococcus mutans, Cronobacter sakazakii, Klebsiella oxytoca and Staphylococcus xylosus) ${ }^{20-21}$. Similarly, the current study also showed that, depending on its mode, RMF exposure increased the growth of G. xylinus by as much as $43 \%$ as compared to the control unexposed cultures. The difference in the number of bacterial cells observed in the current and previous studies can be explained by a much longer cultivation time in the case of the current experiment (the previous study lasted for $6 \mathrm{~h}$, the current study for 6 days). It is worth noting that although the number of G. xylinus cells increased significantly under RMF influence, the number of cellulose non-producing mutants in the exposed cultures remained at the same level as in the control samples.

Table 1. The total number of G. xylinus cells and non-cellulose-producing mutants of G. xylinus in H-S medium and cellulose

\begin{tabular}{|c|c|c|c|c|c|c|c|c|c|c|c|c|}
\hline & \multicolumn{4}{|c|}{ I } & \multicolumn{4}{|c|}{ II } & \multicolumn{4}{|c|}{ III } \\
\hline & \multicolumn{2}{|c|}{$B C$} & \multicolumn{2}{|c|}{ Medium } & \multicolumn{2}{|c|}{$B C$} & \multicolumn{2}{|c|}{ Medium } & \multicolumn{2}{|c|}{$\mathrm{BC}$} & \multicolumn{2}{|c|}{ Medium } \\
\hline & total & mutants & total & mutants & total & mutants & total & mutants & total & mutants & total & mutants \\
\hline RMF exposed & $23 \times 10^{7^{*}}$ & 20 & $29 \times 10^{4}$ & 0 & $30 \times 10^{7 *}$ & 10 & $37 \times 10^{4^{*}}$ & 10 & $20 \times 10^{7^{*}}$ & 0 & $30 \times 10^{4}$ & 10 \\
\hline Control & $18 \times 10^{7}$ & 20 & $29 \times 10^{4}$ & 10 & $21 \times 10^{7}$ & 20 & $26 \times 10^{4}$ & 10 & $17 \times 10^{7}$ & 0 & $31 \times 10^{4}$ & 10 \\
\hline$\%$ of control & $128^{A}$ & - & $100^{\mathrm{a}}$ & - & $143^{\mathrm{B}}$ & - & $142^{b}$ & - & $118^{\mathrm{C}}$ & - & $97^{\mathrm{a}}$ & - \\
\hline
\end{tabular}

Data are presented as a mean number of CFU per $1 \mathrm{~g}$ of cellulose or per $1 \mathrm{~mL}$ medium. * - statistically significant difference between RMF exposed BC and control BC. Mean values with different letters are significantly different (A,B,C for G. xylinus attached to cellulose; $a, b$ for $G$. xylinus present in the medium). The differences were considered statistically significant when the $P$-value was less than 0.05 . 
The process of bacterial cellulose synthesis can be performed in stationary (e.g. horizontal lift reactor, aerosol bioreactor) or agitated conditions using different types of process devices (e.g. stirred tank mixers or reactors, airlift bioreactors, rotary bioreactor, membrane bioreactor $)^{22}$. Regardless of the production method, to obtain a higher $\mathrm{BC}$ yield, a high substrate and oxygen transfer rate are necessary. For this reason, the static culture method is not recommended for mass production $^{23}$. On the other hand, the production of cellulose using shaking or agitated cultivation adjusts oxygen concentration and nutrient supply, but unfortunately also simultaneously has an adverse effect on $G$. xylinus cells through the generated shear forces. Moreover, the shear force generated in shaking/agitated cultivation converts $\mathrm{BC}$-producing strains into cellulose-negative ( $\mathrm{Cel}-$ ) mutants, thereby also resulting in a lower productivity of $\mathrm{BC}^{\mathbf{2 4}}$. Therefore, several production systems were developed to enhance BC production. Yoshino et al. ${ }^{25}$ developed a silicone membrane vessel providing oxygen from the bottom, doubling the rate of $\mathrm{BC}$ production in a cylindrical vessel. Serafica et al. ${ }^{\mathbf{2 6}}$ synthesized bacterial cellulose in a rotating disk bioreactor, which turned out to be more efficient and reduced the time of a run to c.a. 3.5 days instead of the usual 12-20 days. Hornung et al. ${ }^{11}$ developed a reactor in which both glucose and oxygen were directly passed to BC-producing cells. The use of RMF can be considered an intermediate BC production method between stationary and agitated conditions. As a result of using RMF, a pellicle on the surface of the medium similar to that obtained under stationary conditions is produced. However, as demonstrated in our previous studies, the cellulose produced under RMF is characterized by different physical and chemical properties as compared to the material obtained under standard culture conditions (both stationary and agitated $)^{\mathbf{1 4}}$. Besides the direct influence of RMF on the cellulose microfibrils, the additional indirect effect of this kind of magnetic field may also be related with the homogenization of the nutrients and oxygen throughout the culture. Therefore, it can be assumed that the differences observed in the current study in the analyzed biochemical properties (glucose consumption, concentration of acetic acid and gluconic acid) may be attributed to the application of RMF as a factor causing the mixing process and influencing the rate and direction of cell metabolism ${ }^{27}$.

According to Toda et al. ${ }^{28}$ in cultures where glucose is the primary carbon source, high concentration of gluconic acid is the crucial factor responsible for the decreased synthesis of BC. The increased concentration of gluconic acid in the culture medium decreases the $\mathrm{pH}$ of the medium which, in turn, decreases intracellular $\mathrm{pH}$. As a consequence, the high concentration of gluconic acid can change the activity of metabolic pathways responsible for the synthesis of $\mathrm{BC}^{\mathbf{2 4}}$. However, despite differences in gluconic acid content, the $\mathrm{pH}$ of the samples analyzed in the present study (culture medium) remained at a similar level (4.2-4.3) and no significant differences between the various exposure modes were found. So far, the mechanism of the impact of RMF on bacteria cells has not been clearly defined. It was demonstrated that the unique feature of RMF is its ability to induce the time-averaged azimuthal force, which drives the flow of the electrically-conducting fluid in the azimuthal direction $^{29}$. Thus, the RMF acting on the fluid characterized by electrical conductivity can be considered as an alternative method of mixing ${ }^{30-32}$. In magnetic-susceptible liquid (e.g. H-S medium of the electrical conductivity of approx. $7 \mathrm{mS}$ ), an externally applied RMF induces an arbitrary virtual loop which rotates at a frequency equal to the frequency of the supply current. The graphic interpretation of the impact of RMF on the magnetic-susceptible liquid is shown in Figure 5. According to the induction law, the electric current density $J$ is induced along the loop. The interaction between this vector and the magnetic field $B$ generates electromagnetic force $F$ that causes movement of the fluid in the direction of the magnetic field spinning. This force is responsible for the mixing effect, which may increase the efficiency of transport processes between the medium and the microorganisms ${ }^{33-35}$.

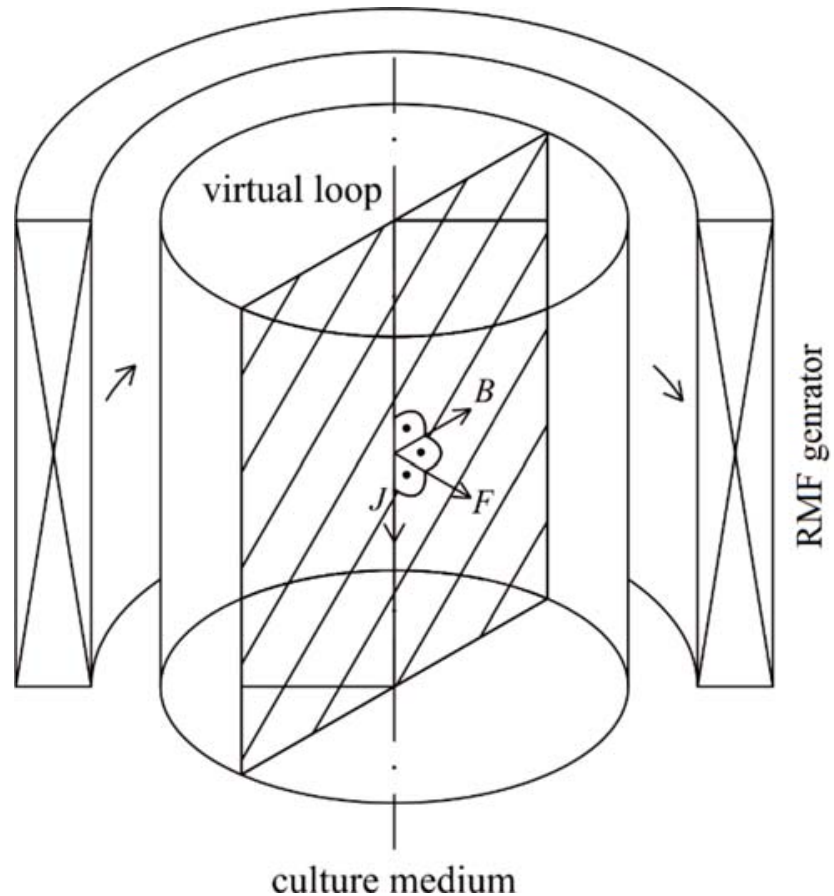

Figure 5. The effect of the RMF on the magnetic susceptible liquid. $\mathrm{B}$ - magnetic induction; F - electromagnetic force; $\mathrm{J}$ - electric current density

In the current study the highest production of gluconic acid in comparison to the unexposed control was found in cultures exposed to RMF using the second mode. These cultures were also characterized by the highest rate of glucose consumption. The synthesis of gluconic acid in bacteria is conducted by membrane-bound glucose dehydrogenase $(\mathrm{GDH})^{\mathbf{2 8}}$. As reported by Zheng et al. ${ }^{\mathbf{3 6}}$ the magnetic field of a specific frequency can increase the activity of this enzyme. Therefore, it can be assumed that the differences in the concentration of gluconic acid observed in the present study could be a result of variances in GDH activity caused by the particular mode of RMF exposure. On the other hand, the second mode of exposure had a simultaneous influence on bacterial growth, which may also explain the increased value of the glucose consumption and higher concentrations of gluconic acid. Such findings are consistent with previous reports from Gao et al. ${ }^{37}$ who reported that the exposu- 
re of bacterial cells to the SMF during various phases of growth of the microorganisms caused differences in expression of the investigated genes. Similarly, Segatore et al. ${ }^{38}$ showed that the effect of exposure of E. coli and Pseudomonas aeruginosa culture to the EMF depended on the age of the analyzed culture. These authors found that the use of EMF in the early phase of microbial growth had a negative impact on the culture's viability. In contrast, the exposure of an older $24 \mathrm{~h}$ culture caused an increase in the number of viable cells. This effect can be explained by the differentiation of the specific metabolic pathways in the bacterial cells depending on the growth phase of the microbial culture ${ }^{39}$.

\section{CONCLUSIONS}

The current study showed that the exposure of the cellulose- synthesizing G. xylinus bacteria to RMF (regardless of the mode) significantly increased the biochemical properties of these microorganisms. The observed effects correlated with the duration and the time of magnetic exposure during G. xylinus cultivation. From the point of view of the efficiency of the $\mathrm{BC}$ production process, the most favourable properties were found for $\mathrm{BC}$ obtained in the setting where the RMF generator was switched off for the first $72 \mathrm{~h}$ of cultivation and switched on for the next $72 \mathrm{~h}$. Moreover, it was proved that RMF had a positive impact on the growth of cellulose-producing bacteria. The magnetic field applied increased the number of G. xylinus cells attached to the cellulose matrix as compared to the unexposed cultures. Furthermore, the application of RMF, regardless of the exposure mode, did not increase the number of generated mutants which were unable to produce cellulose. Our results can be used in subsequent studies concerning the optimization of $\mathrm{BC}$ production using different types of magnetic fields, especially the rotating magnetic field.

\section{ACKNOWLEDGEMENT}

This work was supported by the National Centre for Research and Development in Poland under Grant No. LIDER/011/221/L-5/13/ NCBR/2014.

\section{LITERATURE CITED}

1. Kucińska-Lipka, J., Gubanska, I. \& Janik, H. (2015). Bacterial cellulose in the field of wound healing and regenerative medicine of skin: recent trends and future prospective. Polym. Bull. 72(9), 2399-2419. DOI: 10.1007/s00289-015-1407-3.

2. Ros,s P., Mayer, R. \& Benziman, M. (1991). Cellulose biosynthesis and function in bacteria. Microbiol. Rev. 55(1), 35-58.

3. Koizumi, S., Tomita, Y., Kondo, T. \& Hashimoto, T. (2009). What factors determine hierarchical structure of microbial cellulose - interplay among physics, chemistry and biology. $\mathrm{Ma}$ cromol. Symp. 279(1), 110-118. DOI: 10.1002/masy.200950517.

4. Lei, L., Li, S. \& Gu, Y. (2012). Cellulose synthase complexes: composition and regulation. Front. Plant Sci. 3, 75. DOI: $10.3389 /$ fpls.2012.00075.

5. Ross, P., Weinhouse, H., Aloni, Y., Michaeli, D., Weinberger-Ohana, P., Mayer, R., Braun, S., de Vroom, E., van der Marel, G.A., van Boom, J.H. \& Benziman M. (1987). Regulation of cellulose synthesis in Acetobacter xylinum by cyclic diguanylic acid. Nature 325, 279-281. DOI: 10.1038/325279a0.

6. Yoshinaga, F., Tonouchi, N. \& Watanabe, K. (1997). Research progress in production of bacterial cellulose by aeration and agitation culture and its application as a new industrial material. Biosci. Biotechnol. Biochem. 61(2), 219-224. DOI: 10.1271/bbb.61.219.

7. Li, Y., Tian, C., Tian, H., Zhang, J., He, X., Ping, W. \& Lei, H. (2012). Improvement of bacterial cellulose production by manipulating the metabolic pathways in which ethanol and sodium citrate involved. Appl. Microbiol. Biotechnol. 96(6), 1479-1487. DOI: 10.1007/s00253-012-4242-6.

8. Ragunathan, S. \& Levy, H.R. (1994). Purification and characterization of the NAD-preferring glucose-6-phosphate dehydrogenase from Acetobacter hansenii (Acetobacter xylinum). Arch. Biochem. Biophys. 310(2), 360-366. DOI: 10.1006/ abbi.1994.1179.

9. Yang, X.Y., Huang, C., Guo, H.J., Xiong, L., Luo, J., Wang, B., Chen, X.F., Lin, X.Q. \& Chen, X.D. (2014). Beneficial effect of acetic acid on the xylose utilization and bacterial cellulose production by Gluconacetobacter xylinus. Indian J. Microbiol. 54(3), 268-273. DOI: 10.1007/s12088-014-0450-3.

10. Czaja, W., Romanovicz, D. \& Brown, R.M. (2004). Structural investigations of microbial cellulose produced in stationary and agitated culture. Cellulose 11(3), 403-411. DOI: 10.1023/B:CELL.0000046412.11983.61.

11. Hornung, M., Ludwig, M. \& Schmauder, H.P. (2007). Optimizing the production of bacterial cellulose in surface culture: A novel aerosol bioreactor working on a fed batch principle (Part 3). Eng. Life. Sci. 7(1), 35-41. DOI: 10.1002/ elsc. 200620164.

12. Lin, D., Lopez-Sanchez, P., Li, R. \& Li, Z. (2014). Production of bacterial cellulose by Gluconacetobacter hansenii CGMCC 3917 using only waste beer yeast as nutrient source. Biores. Technol. 151, 113-119. DOI: 10.1016/j. biortech.2013.10.052.

13. Mormino, R. \& Bungay, H. (2003). Composites of bacterial cellulose and paper made with a rotating disk bioreactor. Appl. Microbiol. Biotechnol. 62(5-6), 503-506. DOI: 10.1007/ s00253-003-1377-5.

14. Fijałkowski, K., Żywicka, A., Drozd, R., Niemczyk, A., Junka, A.F., Peitler, D., Kordas, M., Konopacki, M., Szymczyk, P., El-Fray, M. \& Rakoczy, R. (2015). Modification of bacterial cellulose through exposure to the rotating magnetic field. Carboh. Polym. 133, 52-60. DOI: 10.1016/j.carbpol.2015.07.011.

15. Velizarov, S. (1999). Electric and magnetic fields in microbial biotechnology: possibilities, limitations and perspectives. Electro. Magnetobiol. 18(2), 185-212. DOI: 10.3109/15368379909012912.

16. Filipič, J., Kraigher, B., Tepuš, B., Kokol, V. \& MandicMulec, I. (2012). Effects of low-density static magnetic fields on the growth and activities of wastewater bacteria Escherichia coli and Pseudomonas putida. Biores. Technol. 120, 225-232. DOI: 10.1016/j.biortech.2012.06.023.

17. Fojt, L., Strasak, L., Vetterl, V. \& Smarda, J. (2004). Comparison of the low-frequency magnetic field effects on bacteria Escherichia coli, Leclercia adecarboxylata and Staphylococcus aureus. Bioelectrochemistry 63(1-2), 337-341. DOI: 10.1016/j.bioelechem.2003.11.010.

18. Strašák, L., Vetterl, V. \& Fojt, L. (2005). Effects of $50 \mathrm{~Hz}$ magnetic fields on the viability of different bacterial strains. Electromagn. Biol. Med. 24(3), 293-300. DOI: 10.1080/15368370500379715.

19. Hristov, J. \& Perez, V.H. (2011). Critical analysis of data concerning Saccharomyces cerevisiae free-cell proliferations and fermentations assisted by magnetic and electromagnetic fields. Int. Rev. Chem. Eng. 3(1), 3-20.

20. Fijałkowski, K., Nawrotek, P., Struk, M., Kordas, M. \& Rakoczy, R. (2015). Effects of rotating magnetic field exposure on the functional parameters of different species of bacteria. Electromagn. Biol. Med. 34(1), 48-55. DOI: 10.3109/15368378.2013.869754.

21. Fijałkowski, K., Nawrotek, P., Struk, M., Kordas, M. \& Rakoczy, R. (2013). The effect of rotating magnetic field on 
growth rate, cell metabolic activity and biofilm formation by S. aureus and E. coli. J. Magn. 18(3), 289-296. DOI: 10.4283/ JMAG.2013.18.3.289.

22. Lee, K.Y., Buldum, G., Mantalaris, A. \& Bismarck, A. (2014). More than meets the eye in bacterial cellulose: biosynthesis, bioprocessing, and applications in advanced fiber composites. Macromol. Biosci. 14(1), 10-32. DOI: 10.1002/ mabi.201300298.

23. Toyosaki, H., Naritomi, T., Seto, A. \& Yoshinaga, F. (1995). Screening of bacterial cellulose-producing Acetobacter strains suitable for agitated culture. Biosci. Biotech. Biochem. 59(8), 1498-1502. DOI: 10.1271/bbb.59.1498.

24. Park, J.K., Hyun, S.H. \& Jung, J.Y. (2004). Conversion of $G$. hansenii PJK into non-cellulose-producing mutants according to the culture condition. Biotechnol. Bioproc. Eng. 9(5), 383-388. DOI: 10.1007/BF02933062.

25. Yoshino, T., Asakura, T. \& Toda, K. (1996). Cellulose production by Acetobacter pasteurianus on silicon membrane. J. Ferment. Bioeng. 81(1), 32-36. DOI: 10.1016/0922-338X(96)83116-3.

26. Serafica, G., Mormino, R. \& Bungay, H. (2002). Inclusion of solid particle in bacterial cellulose. Appl. Microbiol. Biot. 58(6), 756-760. DOI: 10.1007/s00253-002-0978-8.

27. Morrow, A.C., Dunstan, R.H., King, B.V. \& Roberts, T.K. (2007). Metabolic effects of static magnetic fields on Streptococcus pyogenes. Bioelectromagnetics 28(6), 439-445. DOI: $10.1002 /$ bem. 20332.

28. Toda, K., Asakura, T., Fukaya, M., Entani, E. \& Kawamura, Y. (1997). Cellulose production by acetic acid-resistant Acetobacter xylinum. Ferment. Bioeng. 84(3), 228-231. DOI: 10.1016/S0922-338X(97)82059-4.

29. Rakoczy, R. (2010). Enhancement of solid dissolution process under the influence of rotating magnetic field. Chem. Eng. Process. 49(1), 42-50. DOI: 10.1016/j.cep.2009.11.004.

30. Fraňa, K., Stiller, J. \& Grundmann, R. (2006). Transitional and turbulent flows driven by a rotating magnetic field. Magnetohydrodynamics 42(2-3), 187-197.

31. Walker, J.S. (1999). Models of melt motion, heat transfer and mass transport during crystal growth with strong magnetic field. Prog. Cryst. Growth Ch. 38(1), 195. DOI: 10.1016/S09608974(99)00012-1.

32. Rakoczy, R. \& Masiuk, S. (2010). Influence of transverse rotating magnetic field on enhancement of solid dissolution process. J. AIChE 56(6), 1416-1433. DOI: 10.1002/aic.12097.

33. Moffatt, H.K. (1991). Electromagnetic stirring. Phys. Fluids A 3(5), 1336-1343.

34. Anton-Leberre, V., Haanappel, E., Marsaud, N., Aka, H., Haddour, N. \& Krähenbühl, L. (2010). Exposure to high static of pulsed magnetic fields: does not affect cellular processes in the yeast Saccharomyces cerevisiae. Bioelectromagnetics 31(1), 28-38. DOI: 10.1002/bem.20523.

35. Gaafar, E.S.A., Hanafy, M.S., Tohamy, E.Y. \& Ibahim, M.H. (2008). The effect of electromagnetic field on protein molecular structure of E. coli and its pathogenesis. Rom. J. Biophys. 18(2), 145-169.

36. Zhang, Z., Yang, Z., Zhu, B., Hu, J., Liew, C.W., Zhang, Y., Leopold, J.A., Handy, D.E., Loscalzo, J. \& Stanton, R.C. (2012). Increasing glucose 6-phosphate dehydrogenase activity restores redox balance in vascular endothelial cells exposed to high glucose. PLoS One 7(11). DOI: 10.1371/journal. pone.0049128.

37. Gao, W., Liu, Y., Zhou, J. \& Pan, H. (2005). Effects of a strong static magnetic field on bacterium Shewanella oneidensis: an assessment by using whole genome microarray. Bioelectromagnetics 26(7), 558-563. DOI: 10.1002/bem.20133.

38. Segatore, B., Setacci, D., Bennato, F., Cardigno, R., Amicosante, G. \& Iorio, R. (2012). Evaluations of the effects of extremely low-frequency electromagnetic fields on growth and antibiotic susceptibility of Escherichia coli and Pseudomonas aeruginosa. Int. J. Micro. 7. DOI: 10.1155/2012/587293.
39. Rolfe, M.D., Rice, C.J., Lucchini, S., Pin, C., Thompson, A., Cameron, A.D., Alston, M., Stringer, M.F., Betts, R.P., Baranyi, J., Peck, M.W. \& Hinton, J.C. (2012). Lag phase is a distinct growth phase that prepares bacteria for exponential growth and involves transient metal accumulation. J. Bacteriol. 194(3), 686-701. DOI: 10.1128/JB.06112-11. 